\title{
Erratum: Frustrated Dipole Order Induces Noncollinear Proper Ferrielectricity in Two Dimensions [Phys. Rev. Lett. 123, 067601 (2019)]
}

Ling-Fang Lin, Yang Zhang, Adriana Moreo, Elbio Dagotto, and Shuai Dong®

(Q) (Received 7 November 2019; published 18 December 2019)

DOI: 10.1103/PhysRevLett.123.259901

In our Letter, we studied the noncollinear dipole order in two-dimensional materials $M \mathrm{O}_{2} X_{2}$. There were errors in the notation of the space groups, although the space groups themselves are correct. The only problem is regarding the choice of unique axis. In our figures, the out-of-plane direction is the $c$ axis, while some space groups mentioned in our original Letter correspond to the $b$ axis as the out-of-plane direction.

(i) On page 2 of the main text, the right column, line 9-10: "corresponding to the space groups $C c$ (No. 9) and $P 2{ }_{1} a m$ (No. 26)" should be "corresponding to the space groups $B b$ (No. 9) and $P b 2_{1} m$ (No. 26)."

(ii) In Fig. 2, the notation "P $2_{1} a m$ " should be "Pb2 $2_{1} m$," "Pmma" should be "Pbmm," and "Pmm2" should be "Pm2m."

(iii) The Supplemental Material has also been updated to correct the corresponding issues.

All results and conclusions in our Letter are unaffected.

We thank Professor A. Sundaresan for pointing out the errors. 